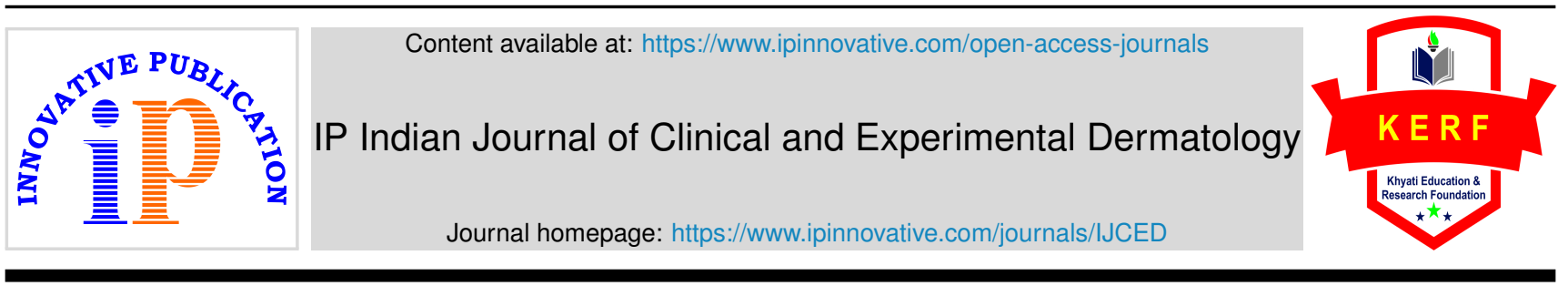

Original Research Article

\title{
Effectiveness and safety of combination of Itraconazole and Amorolfine in management of patients with recalcitrant multi-site dermatophytosis who failed previous combination antifungal therapy
}

\author{
Vishalakshi Vishwanath ${ }^{1}$, Pradnya Londhe ${ }^{1}$, Dakshata Tare ${ }^{1}$, Gaurav Deshmukh ${ }^{2, *}$, \\ Dhiraj Dhoot ${ }^{2}$, Hanmant Barkate ${ }^{3}$ \\ ${ }^{1}$ Dept. of Dermatology, Rajiv Gandhi Medical College \& CSM Hospital, Kalwa, Thane Municipal Corporation, Maharashtra, \\ India \\ ${ }^{2}$ Global Medical Affairs (IF), Glenmark Pharmaceuticals Ltd, Mumbai, Maharashtra, India \\ ${ }^{3}$ Global Medical Affairs (IF \& MEA), Glenmark Pharmaceuticals Ltd, Mumbai, Maharashtra, India
}

\section{A R T I C L E I N F O}

\section{Article history:}

Received 17-11-2020

Accepted 19-11-2020

Available online 05-12-2020

Keywords:

Itraconazole

Amorolfine

Dermatophytosis

Combination Therapy

\begin{abstract}
A B S T R A C T
Introduction: There is alarming rise in cases of dermatophytosis in India. Standard treatment recommendations are no longer working. Combination of topical and systemic antifungal drugs is one of the most frequently used treatment strategy.

Materials and Methods: This was an open label, prospective, interventional, non-comparative study. Effectiveness endpoints were percentage of patients achieving complete cure, clinical cure and mycological cure at the end of the treatment period from baseline. Safety was assessed by analyzing the AEs and monitoring of liver function tests.

Results: 66 patients were included in the study. $75.75 \%, 86.36 \%$ and $81.8 \%$ patients achieved complete cure, clinical cure and mycological cure respectively at the end of the study period. There was improvement in each symptom during study duration as observed during each follow up visit on week 2, 4 and 6 . Total of $10.60 \%$ reported 1 or more drug related AE. Nausea was most common side effect followed by vomiting, headache and edema of face. All the AEs were mild in nature and resolved during study.

Conclusion: To conclude the result of this study shows that the combination therapy of oral itraconazole and topical amorolfine represents an improved treatment strategy for patients with recalcitrant multisite tinea infections.

(C) This is an open access article distributed under the terms of the Creative Commons Attribution License (https://creativecommons.org/licenses/by/4.0/) which permits unrestricted use, distribution, and reproduction in any medium, provided the original author and source are credited.
\end{abstract}

\section{Introduction}

Currently, there is alarming rise in cases of dermatophytosis in India. In current scenario standard treatment recommendations from literature are no longer valid or even realistic. ${ }^{1}$ This has led to practice of experiencebased treatment strategies by majority of dermatologists such as use of higher dose of oral antifungals, prolonged duration of therapy, use of drugs not approved in treatment of dermatophytosis. ${ }^{1,2}$

\footnotetext{
* Corresponding author.

E-mail address: drgaurav0@gmail.com (G. Deshmukh).
}

Combination of topical and systemic antifungal drugs is one of the most frequently used treatment strategy by dermatologists for management of dermatophytosis. It is proving to be more effective than either systemic or topical antifungal alone because of pharmacokinetic advantages of combination therapy. However, there are no definitive comparative studies on combination of systemic and topical antifungal treatment and this strategy is more of hit and trial rather than evidence-based approach. ${ }^{2}$ Thus despite using combination therapy many patients shows recurrence of dermatophytosis. In various studies itraconazole and amorolfine have shown synergistic activity when used in 
combination against dermatophytes. ${ }^{3-5}$

Keeping these scenarios in mind we conducted this study of combination of Itraconazole and Amorolfine because of their proven synergistic activity in management of dermatophytosis in patients who failed to respond to previous combination antifungal therapy or who have developed recurrence despite treatment with combination therapy.

\section{Materials and Methods}

\subsection{Study design}

This was an open label, prospective, interventional, noncomparative, single arm and single center study. The study duration was 6 months from November 2018 to April 2019. The study was approved by Institutional Clinical Ethics Committee (ICEC) and conducted in accordance with the Declaration of Helsinki. The present study is registered at CTRI database (CTRI/2019/04/018560).

\subsection{Patients Selection Criteria}

Patients fulfilling following criteria were included in the study

1. Both male and female patients more than 18 years of age

2. Patients with multi-site tinea infection confirmed by positive $\mathrm{KOH}$ microscopy (Multisite tinea infection was defined as the patients with more than lesions at two different anatomical sites on their body e.g. tinea corporis et cruris meaning patients with tinea lesions on his/her trunk and groin area.)

3. Patients who failed to respond to previous combination therapy or who showed recurrence despite treatment with combination therapy of systemic and topical antifungal agents.

Treatment failure was defined as lack of response after 4 weeks of combination therapy with systemic and topical antifungal drugs. Recurrence was defined as the reappearance of lesions within 6 weeks after completion of the treatment. Combination therapy was defined as simultaneous use of systemic and topical antifungal drugs for management of tinea infections.

Patients with following characteristics were excluded from the study.

1. Patients who received combination of itraconazole and amorolfine for management of tinea infection

2. Patients with single site lesions, concomitant bacterial skin infection, other dermatological conditions like dermatitis, psoriasis, etc.

3. Patients with history of liver or cardiac disease were also excluded from the study
4. Patients receiving immunosuppressive medications or suffering from immunosuppressive illnesses were also excluded from the study.

\subsection{Visits and follow-ups}

After the initial visit, follow-up visits were carried out at 2, 4 and 6 weeks. Those patients who were already taking itraconazole at screening visit were given 1 week wash out period. Each patient was treated with oral Itraconazole $100 \mathrm{mg}$ twice daily for 4 weeks and topical Amorolfine $0.25 \%$ applied twice daily for 6 weeks. During each visit patients were examined for their improvement of symptoms. $\mathrm{KOH}$ examination was performed at initial visit and after 6 weeks. Monitoring of liver function tests was carried for each patient before and during treatment period.

\subsection{Effectiveness Assessment}

\subsubsection{Primary effectiveness end point}

Primary effectiveness endpoint was percentage of patients achieving complete cure at the end of the treatment period from baseline. Complete cure was defined as the patients achieving both clinical cure and mycological cure at the end of treatment.

\subsubsection{Secondary effectiveness end point}

Secondary effectiveness end points were

1. Percentage of patients achieving clinical cure at the end of treatment period. Clinical cure was defined as clear or almost clear symptoms at the end of treatment.

2. Percentage of patients achieving mycological cure at the end of treatment period. Mycological cure was defined as negative microscopy under potassium hydroxide $(\mathrm{KOH})$ examination at the end of therapy.

3. Improvement in each symptom from baseline in each visit.

For effectiveness assessment each patient was evaluated for clinical and mycological improvement. Clinical improvement was assessed by evaluating the improvement in common symptoms of dermatophytosis (scaling, erythema, itching and incrustation) on five-point scale ( $0-$ 4) with 0 being the complete resolution of symptom while 4 being the severe symptom. Mycological improvement was assessed by examining $10 \% \mathrm{KOH}$ mount of skin scraping under microscope before and after treatment.

\subsection{Safety Assessment}

Safety assessment was done by analyzing all the AEs reported by the patients during treatment and by monitoring the liver function tests for evaluating the effect of itraconazole on liver. 


\subsection{Data analysis}

Descriptive statistics were used to summarize effectiveness and safety endpoints using GraphPad Prism version 8 (San Diego, California: GraphPad Software Inc., 20057). P values $\leq 0.05$ were considered statistically significant.

\section{Results}

Among 100 patients screened, 80 patients who fulfilled the inclusion criteria were included in the study. 66 patients completed the follow up period of 8 weeks and were considered for final analysis. 4 patients developed reappearance of lesions during treatment period and discontinued the therapy. 10 patients were lost to follow up.

The average age of the patients was $35.68 \pm 11.32$ years. Out of 66 patients, 36 were male $(54.5 \%)$ while 30 were females $(45.5 \%)$. Tinea corporis et cruris was most common infection $(\mathrm{n}=32,48.4 \%)$. The most common previous therapy was combination oral itraconazole plus topical luliconazole used by $50 \%(n=33)$ of patients. The demographic characteristics of the patients are described in Table 1.

Table 1: Baseline Demographic Details $(n=66)$

\begin{tabular}{|c|c|}
\hline \multicolumn{2}{|l|}{ Variable } \\
\hline Age (Mean, SD) & $\begin{array}{c}35.68 \pm 11.32 \\
\text { years }\end{array}$ \\
\hline \multicolumn{2}{|l|}{ Sex No. $(\%)$} \\
\hline - Male & $36(54.5 \%)$ \\
\hline - Females & $30(45.5 \%)$ \\
\hline \multicolumn{2}{|l|}{ Type of Infection } \\
\hline - Tinea corporis et cruris & $32(48.4 \%)$ \\
\hline - Tinea corporis et cruris et pedis & $16(24.4 \%)$ \\
\hline - Tinea corporis et Cruris et faciei & $6(9.09 \%)$ \\
\hline - Tinea corporis et cruris et pedis et facie & $6(9.09 \%)$ \\
\hline - Tinea cruris et corporis et barbie & $4(6.06 \%)$ \\
\hline - Tinea mannum et cruris et corporis & $2(3.03 \%)$ \\
\hline \multicolumn{2}{|l|}{ Previous Therap } \\
\hline $\begin{array}{l}\text { - Cap. Itraconazole } 100 \mathrm{mg} \text { BD plus } \\
\text { Luliconazole } 1 \% \text { BD }\end{array}$ & $33(50 \%)$ \\
\hline $\begin{array}{l}\text { - Cap. Itraconazole } 100 \mathrm{mg} \text { BD plus } \\
\text { Sertaconazole } 2 \% \text { BD }\end{array}$ & $17(25.75 \%)$ \\
\hline $\begin{array}{l}\text { - Cap. Itraconazole 100mg BD plus } \\
\text { Clotrimazole } 2 \% \text { BD }\end{array}$ & $10(15.15 \%)$ \\
\hline $\begin{array}{l}\text { - T. Terbinafine } 250 \mathrm{mg} \text { BD plus } \\
\text { Luliconazole } 1 \% \mathrm{BD}\end{array}$ & $4(6.06 \%)$ \\
\hline $\begin{array}{l}\text { - T. Griseofulvin 500mg BD plus } \\
\text { Clotrimazole } 2 \% \mathrm{BD}\end{array}$ & $2(3.03 \%)$ \\
\hline \multicolumn{2}{|l|}{ Baseline Symptom Severity (mean, SD) } \\
\hline - Erythema & $2.76 \pm 0.62$ \\
\hline - Scaling & $2.55 \pm 0.73$ \\
\hline - Pruritus & $3.61 \pm 0.52$ \\
\hline - Incrustation & $1.1 \pm 0.81$ \\
\hline
\end{tabular}

\subsection{Effectiveness Analysis}

\subsubsection{Complete cure}

Out of 66 patients analyzed, $75.75 \%(\mathrm{n}=50)$ patients achieved complete cure at the end of treatment period. (Figures 1, 2, 3 and 4) None of the patient who achieved complete cure developed any sign of worsening of symptom after 2 weeks of completion of therapy as confirmed by telephonic follow up.

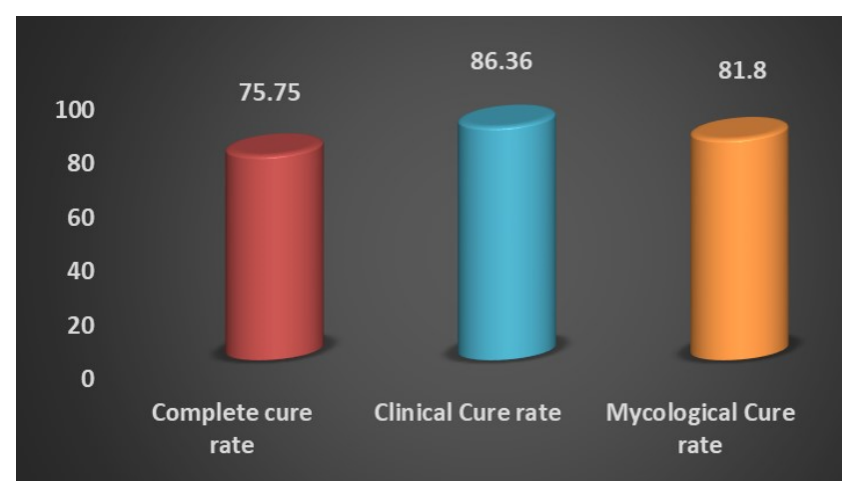

Fig. 1: Effectiveness Assessment of combination of Itraconazole and Amorolfine

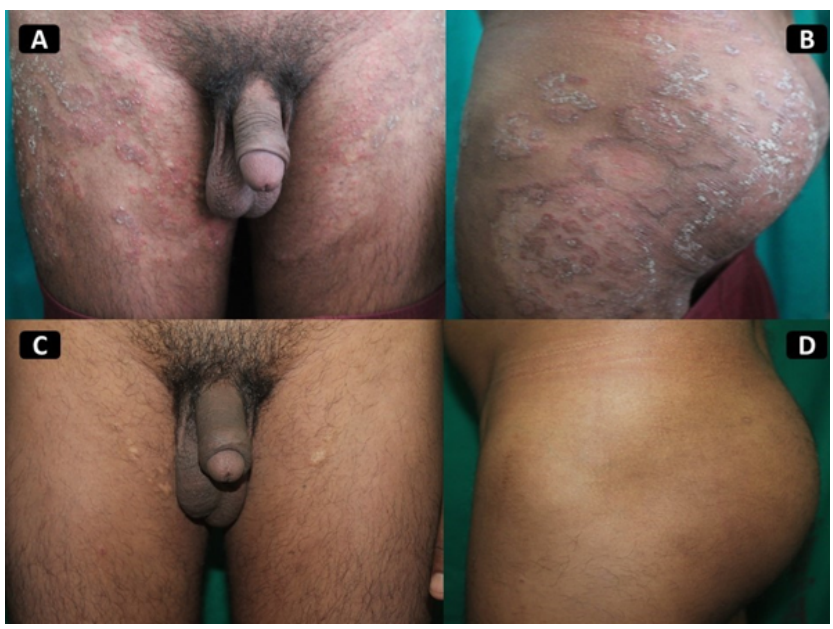

Fig. 2: A, B- Extensive Inflammatory tinea Corporis et cruris with erythema and pustules seen; C, D- Complete resolution of lesions seen at day 42

\subsubsection{Mycological Cure}

At the end of treatment period of 6 weeks, $81.8 \%(n=54)$ patients achieved mycological cure i.e. negative microscopy at the end of treatment period. (Figure 1)

\subsubsection{Clinical Cure and total Symptom score}

After 6 weeks of antifungal therapy, 86.36\% ( $\mathrm{n}=57)$ patients showed complete resolution of their symptoms of erythema, scaling, pruritus and incrustation thus achieving 


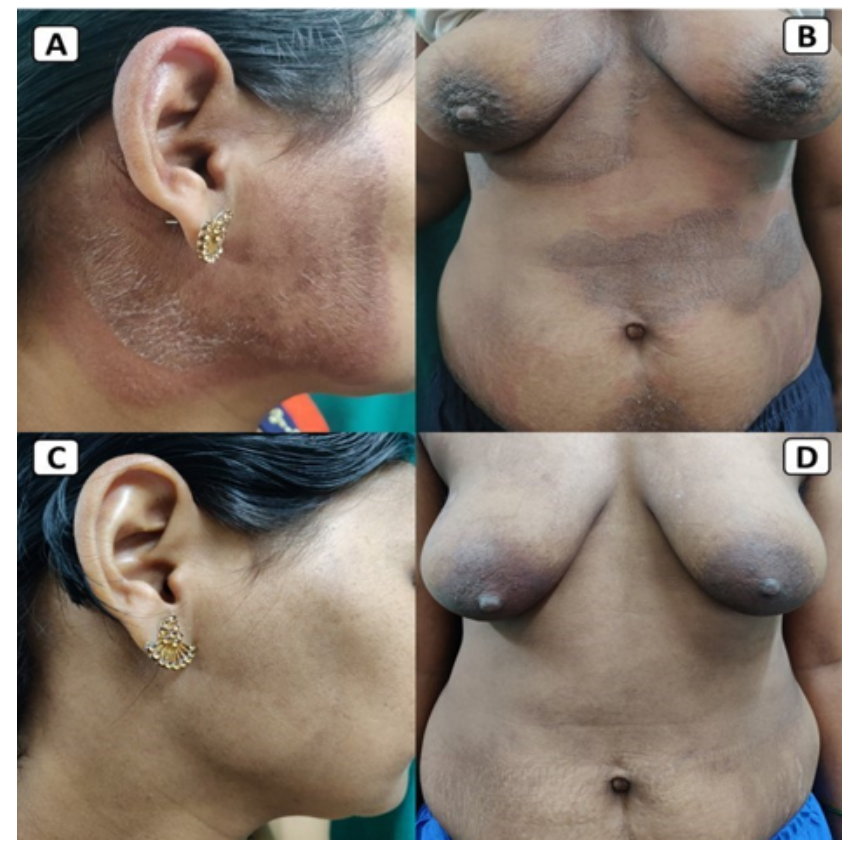

Fig. 3: A, B- Extensive Tinea Corporis et faciei with irritant contact dermatitis; C, D- Complete clearance of lesions in patient with multiple site involvement after 1 month of treatment

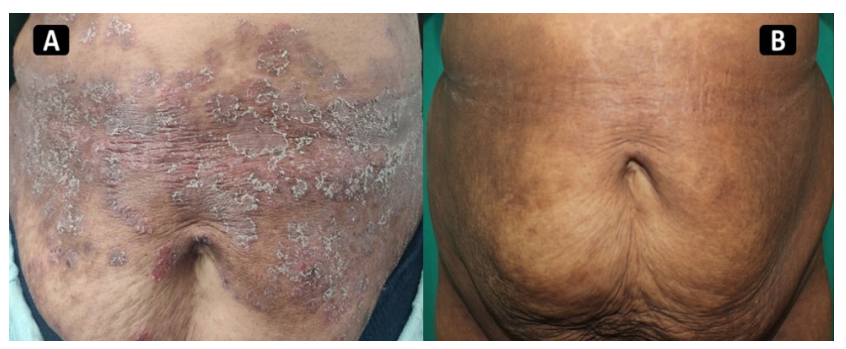

Fig. 4: A- Patient with Tinea Incognito; B- Good clearance of the lesions at end of 1 month

clinical cure (Figure 2). There was improvement in each symptom during study duration as observed during each follow up visit on week 2, 4 and 6. Mean baseline erythema score was $2.76 \pm 0.62$ at the start of study which was significantly decreased to $0.13 \pm 0.33(\mathrm{p}<0.0001)$ after 6 weeks. There was significant improvement in scaling of the patients from baseline at the end of therapy $(2.55 \pm 0.73 \mathrm{vs}$. $0.23 \pm 0.55 ; \mathrm{p}<0.0001)$. Similarly, there was significant decrease in pruritus $(3.61 \pm 0.52$ vs. $0.34 \pm 0.66 ; p<$ $0.0001)$ and incrustation $(1.1 \pm 0.81$ vs. $0.08 \pm 0.28 ; \mathrm{p}<$ 0.0001 ) from baseline at the end of therapy. (Figure 5)

\subsection{Safety analysis}

Out of 66 patients, $10.60 \%(n=7)$ reported 1 or more drug related AE. Nausea was most common side effect reported by $9.09 \%(\mathrm{n}=6)$ patients followed by vomiting $(6.06 \%, \mathrm{n}=$ $4)$; headache $(4.54 \%, \mathrm{n}=3)$ and edema of face $(1.51 \%, \mathrm{n}=$

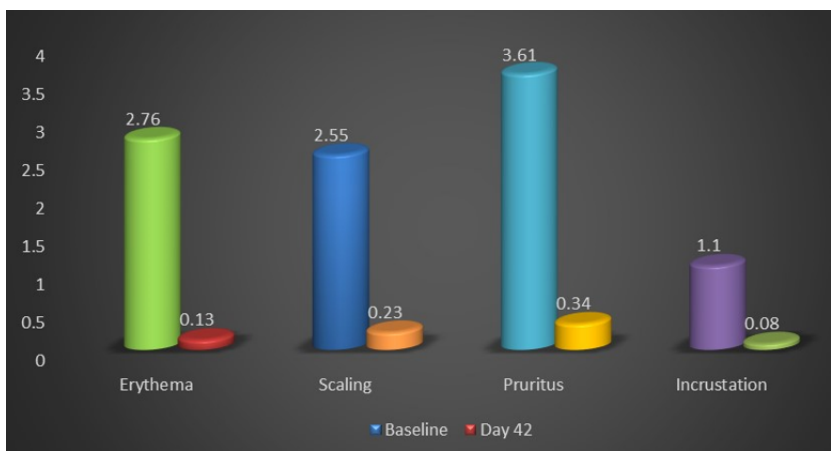

Fig. 5: Improvement in each symptom

1). All the AEs were mild in nature, appeared during initial 2 weeks of therapy and resolved during study, none of the patient discontinued the therapy because of AEs. (Figure 6)

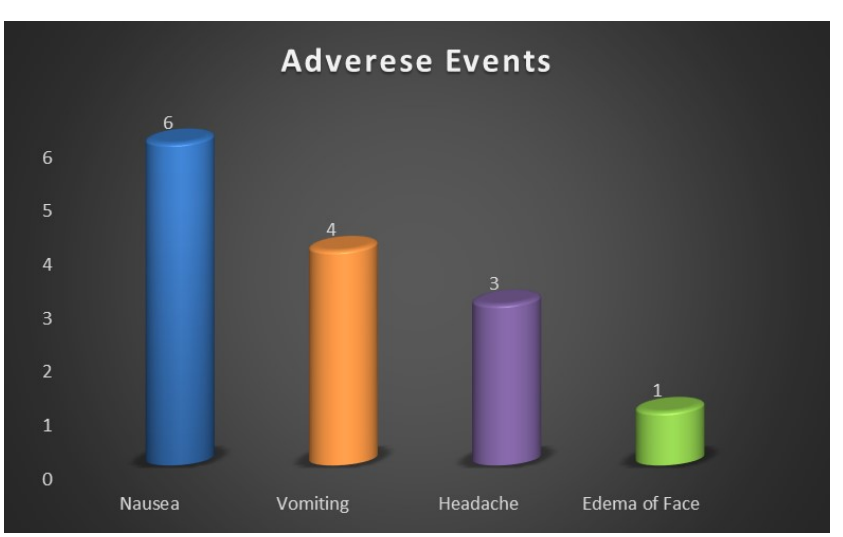

Fig. 6: Adverse events reported by the patients

Liver function of all the patient was evaluated by analyzing the levels of Serum glutamic oxaloacetic transaminase (SGOT) and Serum glutamic pyruvic transaminase (SGPT) before initiation of itraconazole, at week 2 and at week 4 after completion of itraconazole therapy. The baseline SGOT \& SGPT levels were 19.97 \pm 7.34 and $19.86 \pm 11.40$ respectively. After 2 weeks of itraconazole therapy there was no significant change in SGOT and SGPT levels $(19.06 \pm 7.44$ and $18.62 \pm 10.70$ respectively, $\mathrm{p}=0.1)$. At week 4 similar results were seen with no significant change in SGOT and SGPT levels (19.76 \pm 7.40 and $19.02 \pm 11.50$ respectively, $\mathrm{p}=0.1$ ).

\section{Discussion}

Based on initial studies and recommendations topical antifungals are first line drugs in management of tinea infections. However, in patients with large lesions or multisite tinea infections only topical therapy fails to clear lesions leading to treatment failures. Systemic therapy is recommended in such patients where topical therapy fails. ${ }^{6}$ 
Oral itraconazole is drug of first choice in management of tinea infections in current scenario in India. Various earlier studies have shown variable but appreciable cure rates for dermatophytosis in the range of 72.7-96.6\% following itraconazole therapy. ${ }^{7-11}$ However recently lower cure rates with itraconazole have been reported. Sharma et al in their study reported only $50 \%$ cure rate with 3 weeks $200 \mathrm{mg}$ /day itraconazole therapy. ${ }^{12}$ Similar decrease in clinical efficacy of amorolfine is evident from recent report by Das et al who reported only $26.09 \%$ complete cure rate with amorolfine which was lower as compared to previous studies showing variable cure rates of $80-90 \% .^{13}$

Hence combination therapy seems more useful in management of patients with multi-site tinea infections. Sahoo et al and Murlidhar et al in the comprehensive reviews recommended the use of combination of topical and systemic antifungals in management of patients with large lesions or recalcitrant tinea infections. Authors commented that while using combination therapy drugs from two different class should be used for wider coverage, synergistic or additive action and to reduce the chance of resistance. ${ }^{6,14}$

In our study $90.0 \%(\mathrm{n}=60)$ patients were previously treated with combination of oral and topical azole. The use of combination of same class of drugs might be one of the reasons of treatment failure seen in these patients. Various in-vitro studies have demonstrated mixed results of antagonism or indifference with combination of two drugs acting at same target. ${ }^{15,16}$

Synergistic activity of combination of itraconazole and amorolfine has been shown in some in-vitro studies. ${ }^{3,4}$ In humans the improved activity of combination has been shown in patients with onychomycosis where combination therapy was better than monotherapy with itraconazole. ${ }^{5}$ Theoretically sequential inhibition of $14 \alpha$ demethylase by itraconazole and inhibition of delta-14-reductase and delta-7, 8-isomerase by amorolfine may result in more complete suppression of ergosterol synthesis resulting better antifungal activity.

In present study complete cure rate of $75.75 \%$ was more compared to monotherapy with itraconazole $(50 \%)$ or amorolfine $(26.09 \%)$ alone. Mycological cure rate in our study was $81.8 \%$ which is higher than the mycological cure rate reported for monotherapy with either drug. Das et al in their study reported mycological cure rate of $80 \%$ while Banerjee et al reported mycological cure rate of $78.9 \%$ in patients treated with amorolfine. ${ }^{13,17}$ Similarly Sharma et al in their study reported mycological cure rate of $50 \%$ for itraconazole monotherapy. ${ }^{12}$ This clearly indicates that the combination therapy with amorolfine and itraconazole is better than either therapy alone in management of recalcitrant multisite tinea infection.

Similarly, the clinical cure rate in our study was better than the clinical cure reported for either drug alone. In our study $86.36 \%$ patients achieved clinical cure rate compared to clinical cure rate of $50 \%$ reported by Sharma et al in their study for itraconazole monotherapy. ${ }^{12}$

With regards to safety, only $10.60 \%$ patients reported 1 or more AE in our study. All the adverse effects were of mild to moderate severity and none of the patients required discontinuation of therapy. None of the patient in our study reported any derangement of liver function during treatment period. Adherence to treatment was excellent in all the patients. These results are in accordance with the safety profile of both amorolfine and itraconazole reported in earlier studies. ${ }^{7-11}$

\section{Limitations}

Limitations of our study include the small population. The fungal culture and sensitivity could not be done due to nonfeasibility.

\section{Conclusion}

To our knowledge this is the first Indian study providing the evidence that the commonly used treatment strategy of combination of topical and systemic antifungal drugs is efficacious and safe in management of multi-site tinea infections. This study also proves that use of combination of two different class of antifungal drugs is associated with improved antifungal activity.

To conclude the result of this study shows that the combination therapy of oral itraconazole and topical amorolfine represents an improved treatment strategy for patients with recalcitrant multisite tinea infections who failed previous combination antifungal therapy.

\section{Source of Funding}

No financial support was received for the work within this manuscript.

\section{Conflict of Interest}

The authors declare they have no conflict of interest.

\section{References}

1. Verma S, Madhu R. The Great Indian Epidemic of Superficial Dermatophytosis: An Appraisal. Indian J Dermatol. 2017;62(3):22736.

2. Dogra S, Uprety S. The menace of chronic and recurrent dermatophytosis in India: Is the problem deeper than we perceive? Indian Dermatol Online J. 2016;7(2):73-6. ब01:0.410312222 D.78. 18100

3. Tamura T, Asahara M, Yamamoto M, Yamaura M, Matsumura M, Goto K, et al. In vitrosusceptibility of dermatomycoses agents to six antifungal drugs and evaluation by fractional inhibitory concentration index of combined effects of amorolfine and itraconazole in dermatophytes. Microbiol Immunol. 2014;58(1):1-8.

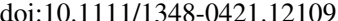

4. Lecha M. Amorolfine and itraconazole combination for severe toenail onychomycosis; results of an open randomized trial in Spain. $\mathrm{Br} J$ Dermatol. 2001;145:21-6. 
5. Lesher JL. Oral therapy of common superficial fungal infections of the skin. $\quad J$ Am Academy Dermatol. 1999;40(6):S31-4. do1:10.1016/s0190-9622(99)70395-6.

6. Mahajan R, Sahoo AK. Management of tinea corporis, tinea cruris, and tinea pedis: A comprehensive review. Indian Dermatol Online J. 2016;7(2):77-86. 101:104103/222प-5 /8. ग1800प

7. Boonk W, Geer DD, Kreek ED, Remme J, Huystee BV. Itraconazole in the treatment of tinea corporis and tinea cruris: comparison of two treatment schedules. Mycoses. 1998;41:509-14.

8. Katsambas A, Antoniou C, FrangouFli E, Rigopoulos D, Vlachou $\mathrm{M}$, Michailidis D, et al. Itraconazole in the treatment of tinea corporis and tinea cruris. Clin Exp Dermatol. 1993;18(4):322-5. do1:10.1111/.1365-2230.1993.tb02207.x.

9. Acharya KM, Mukhopadhyay A, Thakur RK, Mehta T, Bhuptani $\mathrm{N}$, Patel R. Itraconazole versus griseofulvine in the treatment of tinea corporis and tinea cruris. Indian J Dermatol Venereol Leprol. 1995;61:209-11.

10. Keyser PD, Backer MD, Massart DL, Westelinck KJ. Twoweek oral treatment of tinea pedis, comparing terbinafine (250 $\mathrm{mg}$ /day) with itraconazole (100 mg/day): a double-blind, multicentre study. Br J Dermatol. 1994;130(s43):22-5. d01:10.1111/13.1365-

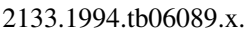

11. Bourlond A, Lachapelle JM, Aussems J, Boyden B, Campaert $\mathrm{H}$, Conincx S, et al. Double-Blind Comparison of Itraconazole with Griseofulvin in the Treatment of Tinea Corporis and Tinea Cruris. Int J Dermatol. 1989;28(6):410-12. ॠoi:10.111/].136543621080 th02401x

12. Sharma P, Bhalla M, Thami GP, Chander J. Evaluation of efficacy and safety of oral terbinafine and itraconazole combination therapy in the management of dermatophytosis. J Dermatol Treat. 2020;31(7):74953. 10:-101080/00546634.201016/2835.

13. Sil A, Das A, Sarkar TK, Sen A, Chakravorty S, Sengupta M. A randomized, double-blind trial of amorolfine $0.25 \%$ cream and sertaconazole $2 \%$ cream in limited dermatophytosis. Indian J Dermatol, Venereol Leprol. 2019;85:276-81. do1:10.4I03/1]dvl.1]dv1_90/_T7.

14. Rajagopalan M, Inamadar A, Mittal A, Miskeen AK, Srinivas CR, Sardana K, et al. Expert Consensus on The Management of
Dermatophytosis in India (ECTODERM India). BMC Dermatol.

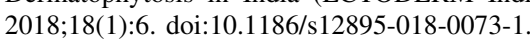

15. Harman S, Ashbee HR, Evans EGV. Testing of antifungal combinations against yeasts and dermatophytes. J Dermatol Treat 2004;15(2):104-7. 10:-10.1080/09546630410025988

16. Johnson MD, Perfect JR. Use of Antifungal Combination Therapy: Agents, Order, and Timing. Curr Fungal Infect Rep. 2010;4(2):87-

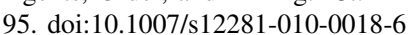

17. Banerjee M, Basak S, Gangopadhyay DN, Ghosh AK, Das KD Comparative evaluation of effectivity and safety of topical amorolfine and clotrimazole in the treatment of tinea corporis. Indian J Dermatol. 2011;56(6):657-662. 10:10.4103/0010-515491823.

\section{Author biography}

Vishalakshi Vishwanath, Professor and Head

Pradnya Londhe, Associate Professor

Dakshata Tare, Assistant Professor

Gaurav Deshmukh, Manager

Dhiraj Dhoot, Senior Manager

Hanmant Barkate, Vice President

Cite this article: Vishwanath V, Londhe P, Tare D, Deshmukh G, Dhoot $\mathrm{D}$, Barkate $\mathrm{H}$. Effectiveness and safety of combination of Itraconazole and Amorolfine in management of patients with recalcitrant multi-site dermatophytosis who failed previous combination antifungal therapy. IP Indian J Clin Exp Dermatol 2020;6(4):391-396. 